\title{
Visualizing and Understanding ATLAS Calorimeters with Atlantis
}

\author{
A.A Nepomuceno * \\ Federal University of Rio de Janeiro \\ for the ATLAS Collaboration \\ E-mail: andre.asevedodcern.ch
}

We describe tools that have been developed for the visualization and understanding of the hadronic and electromagnetic calorimeters of the ATLAS experiment with the event visualization program Atlantis. The event data is extracted into XML format by dedicated algorithms running within the ATLAS Software framework ATHENA, and read into Atlantis directly from physical datafiles or via a server/client-setup. For the ATLAS Calorimeters commissioning, code was developed to create XML-data with detailed calorimeter information, like the electronic pulse shapes, calibration factors and cell identifiers. All this information is displayed in Atlantis through tools developed for this purpose. With these new features, the user has access to the reconstructed and raw data in the same place, and can check for example the consistency of the reconstruction. It allows easy identification of problematic calorimeter cells, e.g. high noise or dead cells, and also allows one to compare digital data from calorimeter and trigger channels, and was used during the ATLAS detector commissioning in 2008 and 2009.

XXth Hadron Collider Physics Symposium

November 16 - 20, 2009

Evian, France

${ }^{*}$ As well as the author, the developers directly involved in the work described are Z. Maxa, S. Boeser and R. Teuscher. We would like to thank the Atlantis developer team for their support: Adam Davison, Charles Timmermans, Eric Jansen, Juergen Thomas, Mark Stockton, Nikos Konstantinidis, Peter Klok and Peter Watkins. We would also like to thank the Tile and LAr calorimeter experts for discussion and help, in particular Sanya Solodkov, Luca Fiorini, Guillaume Unal, Adam Gibson and Bin Guo. A.A Nepomuceno thanks the conference for the financial support. 


\section{ATLAS Calorimeters}

The ATLAS calorimeters consist of an electromagnetic (EM) calorimeter and a hadronic calorimeter [1] that use two technologies: liquid argon and scintillating tiles. Liquid argon is used for the EM calorimeter, hadronic end-cap and forward calorimeter, while scintillating tiles are used for the barrel and extended barrel hadronic calorimeter. These calorimeters cover the range of $|\eta|<4$.9. A view of the ATLAS calorimeters is shown in Figure 1.

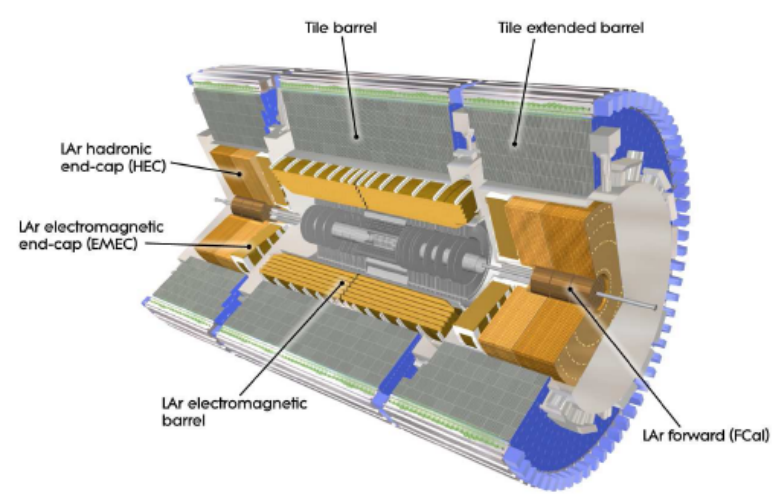

Figure 1: ATLAS Calorimeters

\section{The ATLAS Software}

The ATLAS software, Athena, is organized in a hierarchical structure of packages that include specific algorithms for each subdetector, generator, trigger, reconstruction, software version and so on. When the raw or simulated data are reconstructed, the following data files are produced:

Event Summary Data (ESD) - ESD refers to event data written as the output of the reconstruction process. Its content is intended to make access to raw data unnecessary for limited calibration or re-reconstruction. It is stored in POOL ROOT files.

Analysis Object Data (AOD) - AOD is a reduced event representation, derived from ESD, suitable for physics analysis. It contains physics objects and it is stored in POOL ROOT files.

\section{Atlantis Event Display}

Atlantis a is stand-alone Java application with the primary goal of the visual investigation and understanding of the physics of the complete event. It also helps in the development and debugging of reconstruction software and data quality during detector commissioning. In order to display an event in Atlantis, the event data must be converted to XML-format. This task is performed by an Athena package called JiveXML. Event files used as input for JiveXML can either be raw data or reconstructed ESD/AOD files. The event data is accessed from Atlantis in two ways: using the event files produced by JiveXML (offline mode); or reading the event data over network from JiveXML server (online mode). 


\section{Atlantis For Calorimeter Commissioning}

Specific tools have been developed to help in data debugging for ATLAS combined calorimeter commissioning. These tools consist of a package integrated into Athena called CaloJiveXML. It has a series of code written in C++ that creates XML-data with detailed calorimeter information. The information is produced by calorimeter reconstruction algorithms, posted to a common in-memory database, and then retrieved by CaloJiveXML to create the XML files. The package includes a dedicated retriever for each calorimeter and for the Minimum Bias Trigger Scintillators (MBTS).

The calorimeter information available in the XML file is displayed in Atlantis through tools developed with the Java Analysis Studio package. In a very easy and fast way, the user has access to detailed calorimeter cell data like energy and time for each channel, cell identifier, channel gain, channel pedestal, channel reconstruction quality and raw data and predicted pulse shape for each channel.

With these new features, Atlantis displays reconstructed and raw data in the same place, which allows the user to check the consistency of the reconstruction and the algorithms performance. It also helps in the easy identification of problematic cells, like high noise or dead cells, and the identification of sources of fake missing transverse energy.

Figures 2 shows the display of a collimator splash event seen by ATLAS. The red colour indicates the Tile and End-cap hadronic calorimeters, the green represents the electromagnetic and forward calorimeters and the blue the muon system. The yellow rectangles are the cells where energy has been deposited.

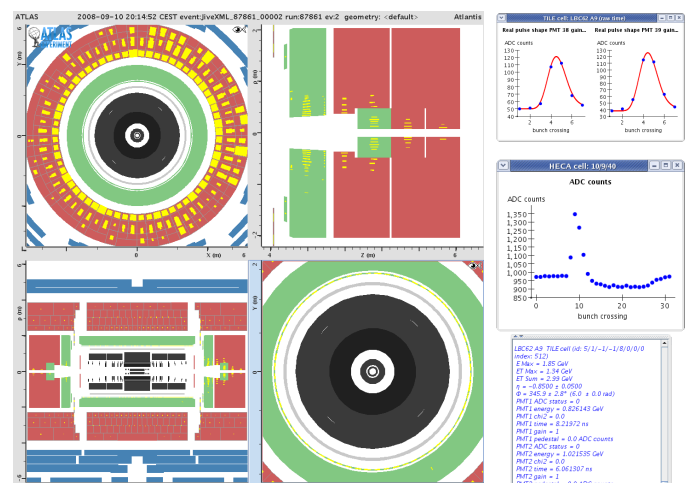

Figure 2: Atlantis Event Display for a splash event seen by ATLAS

In the plots above, the blue dots are the digitized pulse shape and the red curve is the predicted pulse shape for the tile channel. The detailed cell information is shown on the bottom right hand of the display. It is used for checking electronics problems, pulse shape models and also for checking the time of flight in Tile calorimeter data. The tools developed have been extensively used in different phases of the calorimeter commissioning, helping to find and solve various problems.

\section{References}

[1] The ATLAS Collaboration, G. Aad et al., JINST 3 S08003 (2008). 